\title{
A Study on Character Recognition of Korean Vehicle License Plates Based on Deep Learning
}

\author{
Yongjun Kim, Wooyoung Soh \\ Graduate School of Computer Engineering, Hannam University \\ yjkim0123@gmail.com,wsoh@hnu.kr
}

\begin{abstract}
In this paper, a study was conducted on the improvement of the recognition rate for numbers and Korean characters (Korean characters) that were unclear due to partial contamination or damage of Korean license plates using CNN deep learning algorithm.

In this paper, research and experiments were conducted with several constraints. The subject of the study is not normal characters, but partially damaged and unclear characters due to contamination, and the purpose of these characters is to improve the recognition rate using $\mathrm{CNN}$ deep learning algorithm. The training data used in this paper, the shape (degree of damage) and number of actual data, and the number of training were arbitrarily limited.

In addition, there is a difference in the recognition rate according to the degree of contamination and damage and uncertainty of each numeric data. The overall recognition rate for numeric characters is $80 \%$ and the recognition rate for Korean characters is $76.5 \%$. Perhaps, if the training data is input more diversely and trained, the recognition rate will be improved a little more.

As a result of the experiment, there was a slight difference in recognition rate between numbers and Hangul (Korean characters), and there was a slight difference in recognition rate according to the degree of contamination and damage.
\end{abstract}

Keywords: Deep learning, character recognition, vehicle license plates, cnn, Korean characters. 


\section{Introduction}

Now that traffic volume is rapidly increasing due to industrial development and economic growth, many methods and systems for vehicle management are being proposed, but manpower waste and difficulties in management remain. Therefore, it is urgent to develop an automatic license plate recognition system for parking lot access control and arrested vehicles according to the requirements of various fields. Research in this field is currently being conducted mainly by several universities or private companies, and related thesis is also in the academic world. Was announced (Choi, 1993; Kim and Choi, 1995).

The following Figure 1 shows the changes in Korean license plates. The most important process to be solved for the recognition of the license plate is the process of accurately extracting the characters inside the license plate from the input image. However, it is not easy to effectively separate and extract each number because the numbers in the serial number are connected to each other while going through the process of binarization by miscellaneous goods or the number plate decorations that are currently used a lot.

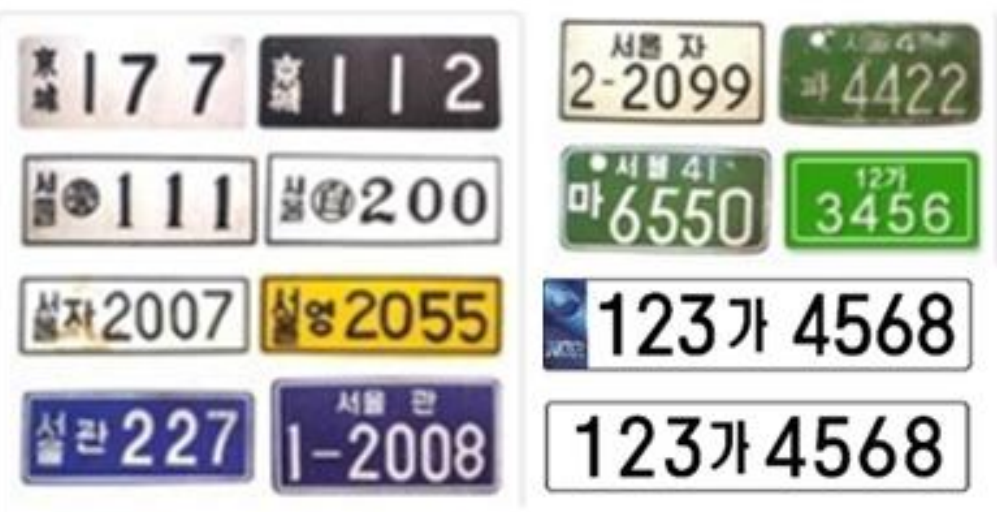

Fig. 1: Changes in Korean vehicle license plates.

These days, the parking management system of apartments consists of a large part of the automatic switch, and the complaints of the apartment management service increase due to frequent fertilization due to some recognition errors, and the reliability of the management office and the apartment parking system is also falling a lot. In addition, the amount of work of unnecessary security guards or officials is increased through this, resulting in waste of labor costs.

Therefore, the purpose of this study is to minimize such inconvenience, and to significantly increase the efficiency of recognition by applying CNN deep learning to recognition using existing image processing. 


\section{Research Method and Scope}

The parking management system, which is currently generally used, is maintained as it is, and research is conducted to improve the recognition rate of such an existing system.

In the case of most unclear Hangul (Korean characters) letters and numbers that are difficult to recognize, there are many differences depending on the degree of distortion, damage, and contamination of the characters. It is to conduct research to significantly improve the recognition rate through the improved recognition used.

Basically, by performing deep learning training for an appropriate number of times with the prepared training data, and by repeatedly recognizing the randomly contaminated experimental data prepared in advance, the recognition rate for distorted or damaged and contaminated characters (Hangul or numbers) in various situations. We intend to conduct a study to measure and see the results of improving recognition rate. Several steps are required for this experiment. First, 112 letters of various types were prepared for training numbers and Hangul data, and 11 times for numbers and 10 times for letters were studied. In addition, 30 pieces of data to be used for the actual test were prepared and damaged and contaminated at random, and this is an experimental stage to determine the recognition rate (accuracy) through this algorithm (Kim, 2020).

For this experiment, research and experiments were conducted with the following research scope and some constraints (Kim, 2020).

(1) A study on the recognition of automobile license plates in Korea

(2) A study on the recognition of license plates that are partially damaged and unclear due to contamination other than normal characters

(3) The shape (degree of damage) and number of training data and actual data, and the number of training are arbitrarily limited for this experiment.

(4) The area of research on automobile recognition is limited to areas where apartments and other vehicles are registered and managed.

(5) The core of this study was limited to research on the recognition of unclear characters (numbers and Korean characters) based on CNN deep learning algorithm using Python language.

\section{Related Research}

\subsection{Deep Learning}

Deep learning is a technique used to cluster or classify objects or data. Computers, for example, cannot differentiate between dogs and cats only by pictures. However, humans can be distinguished very easily. For this, a method called 'Machine Learning' was devised. It is a technology that allows you to input a lot of data into a computer and sort similar ones. When a picture similar to a saved dog picture is input, the computer classifies it as a dog picture. Many machine learning algorithms 
have already emerged as to how to classify data (Naver Encyclopedia of Knowledge, 2020).

Research on deep learning has been studied in various forms. Applications include research on image restoration, research on text using big data, and research on speech recognition. The end of deep learning is also varied, and there are deep learning algorithms based on YOLO, CNN, and GAN (Weon and Lee, 2019; Seo and Kim, 2016; Zhang, 2018).

It is an advanced form of machine learning that learns and predicts by itself without requiring humans to input data to learn. This model was developed from an artificial neural network modeled after a human neural network. Deep learning is one of the machine learning techniques, Feature Learning (or Representation Learning). Deep learning is a deep neural network (DNN) developed from an artificial neural network. Deep learning is called hierarchical Feature Learning (or Representation Learning) because it is a model of a method in which several nerves transmit signals to the next nerve (Kang, 2020).

$\mathrm{CNN}$ (convolution neural network), which is widely used in computer vision, consists of an input layer, an output layer, and several hidden layers. Since convolution calculations are performed in the hidden layer, this neural network is called a CNN. It is also called convolutional neuron networks, or ConvNets. This neural network, which had already appeared at the end of the 90s, aroused a lot of interest as deep learning developed, showing surprising results in the field of image recognition. The main difference from the commonly known neural network structure is that the $\mathrm{CNN}$ input is a picture (= image). And it captures the features in the picture (Sai, 2020).

\subsection{Character Recognition and Image Recognition}

Automatic recognition of letters or numbers is used in many fields today. In particular, it is widely used in metering systems and vehicle license plate recognition. There are several approaches to character or number recognition, such as circular matching method, morphological analysis method, feature-based method, and statistical analysis method (Shang and Brown, 1994; Shon, 2003). Alphanumeric recognition can be largely divided into printed number recognition and handwritten number recognition. This paper aims to recognize printed letters and numbers (Liu and Kim, 2015).

On the other hand, letters and numbers have structural features. Based on these structural features, Z. Zhang et al. used shape feature information as a recognition method for reading numbers of meter devices. In the case of numbers, the objects from 0 to 9 were divided into a closed circle and a half-closed circle, and a vertical line was drawn for each number to distinguish the number based on the intersection point (Sai, 2020).

CNN (Convolutional Neural Network) began in the late 1990s as research on 
handwriting recognition. It is a model suitable for image processing by implementing human visual processing. As a result of recent research, the accuracy of the model has been increased by increasing the layers of the model, especially in the field of image recognition (Bae, 2020; Kim et al., 2019).

RNN (Recurrent Neural Network) is a model used for sequential data processing, and shows excellent performance in sequentially extracting data characteristics by recursively using intermediate layer values of deep neural networks. Currently, models such as LSTM (Long Short Term Memory) and GRU (Gated Recurrent Unit) have been developed and are used for speech recognition and natural language processing (Macfee, 2017).

\subsection{Korean Car License Plate Recognition}

Looking at the existing general vehicle number recognition flow chart is as follows. First, in the case of a parking lot, a loop is installed in front of a circuit breaker, and when a vehicle enters the parking lot, a photo is taken to obtain an image when a loop signal is received, and in the case of a high pass installation area, images are taken at short intervals. Image quality, color, and noise are improved on the captured image, and the license plate part is inferred from the image acquired from the camera through the edge detection method (Lee, 2017).

The Korean license plate consists of 2 to 3 numbers, 1 Hangul (Korean character), and 4 numbers. Hangul characters are one of 74 Hangul characters. Each Hangul character consists of a combination of one consonant and one vowel. Korean consonants are 'フ', '', '', 'ㄹ', 'ㅁ', 'ㅂ', '入', 'O', 'ㅈ', and ''. and It corresponds to 'k', 'n', 'd', 'l', 'm', 'b', 's', 'o', 'g', and 'h'. There are 4 Korean vowels, 'ト', '才', '上', and '丁', which correspond to 'a', 'eo', 'o', and 'u' (Tang et al., 2019).

\section{Damaged Character Recognition of Korean License Plate Based on Deep Learning}

Several steps are required for this experiment. First, 112 letters of various types were prepared for training numbers and Hangul data, and 11 times for numbers and 10 times for letters were studied. In addition, 30 pieces of data to be used in the actual test were prepared and damaged and contaminated arbitrarily, and through this, the recognition rate (accuracy) is determined through this algorithm. The Python language (Python 2.7+) was used for the implementation of this study.

\subsection{Experiment with CNN Deep Learning Algorithm}

This study was conducted by applying CNN deep learning algorithm, and experiments were conducted for deep learning of numbers and some characters (anaconda opensource, 2019). In the code below, it is difficult to obtain enough data for training from the original data, so it is a function that increases the amount of data through steps such as rotating or distorting, and the process of generating 30 
different data per data. The following Figure 2 is the coding contents of the Python program implementing this.

The next Figure 3 is a part of designating the location of the folder to load data for training and testing, and designating the size and class to designate where each data will be placed.

First, it shows the process of loading each folder containing the training object and the test object. This is the process of loading 4026 images in 10 classes from 0 to 9 , which are $28 * 28$ sized to train numbers, and test the actual numbers. It shows the process of loading 300 images in 10 classes ranging from 0 to 9 , which is $28 * 28$ size to do.

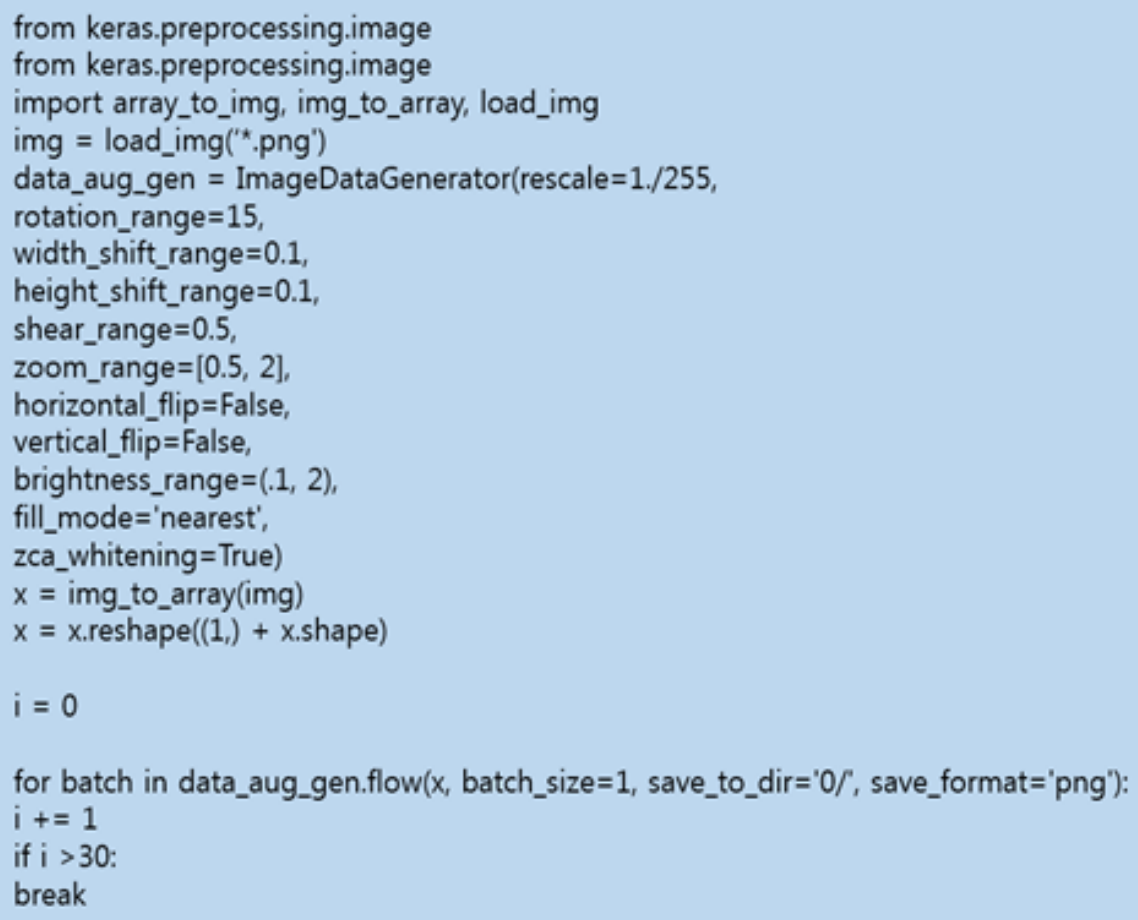

Fig. 2: Implementation coding for generating 30 different data per data. 


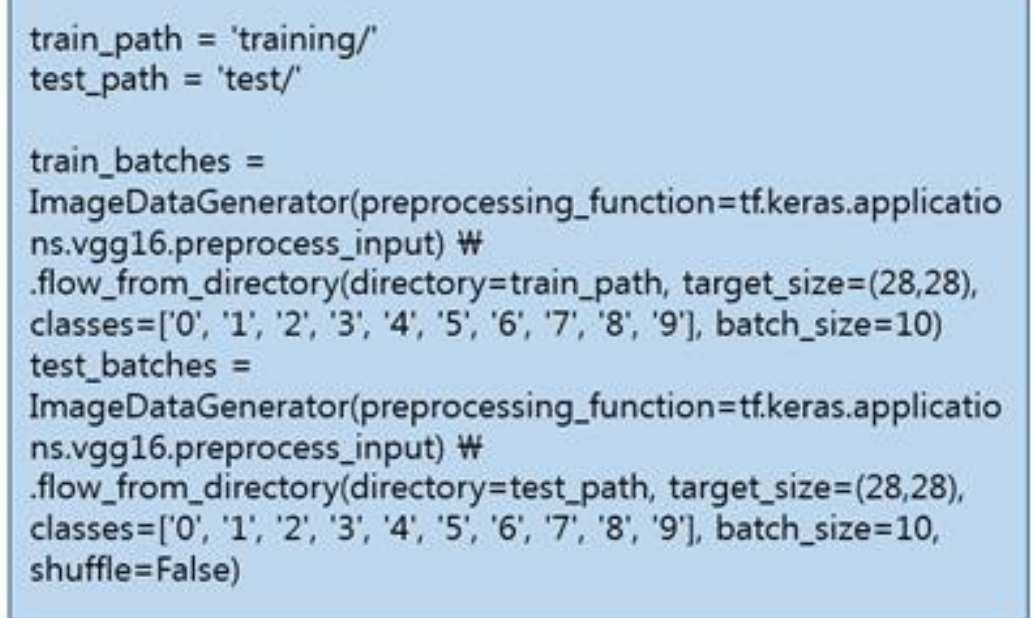

Fig. 3: Size and class implementation coding for data storage.

In addition, the part that loads 820 images in four classes of $28 * 28$ size for training Hangeul, "가", “라", “마", and “자", and 28*28 size for actually testing Hangeul, Have the 120 images in the class load.

And the training image is loaded and shown, and the image is composed of numbers and letters. In the case of numbers, it is 10 example data from " 0 " to " 9 ". It was tested with the example data of.

The following Figure 4 shows the results of numbers (0 9) and letters ("가") in the test image.

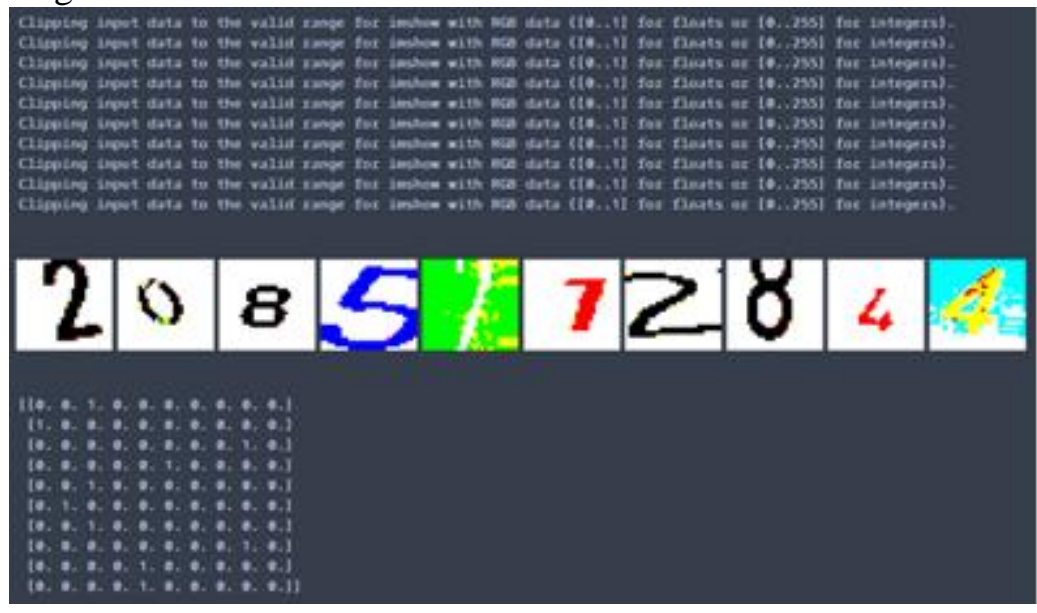




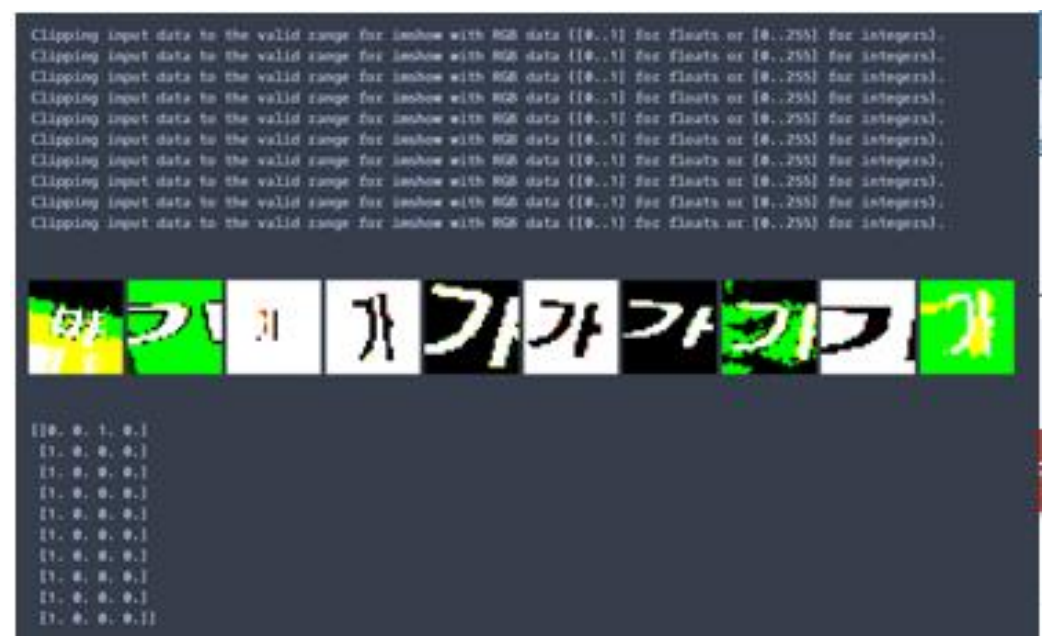

Fig. 4: Results of Numbers (0 9) and Korean character (가) in the test image.

\subsection{Numeric and Korean Character Data for Training and Testing [4]} 112 data were prepared for each character of the training data used for this study, and the actual data assigned to the actual CNN deep learning were tested by preparing 30 characters each as follows.

Figure 5 shows examples of objects to be trained (" 0 ", “가"”), and the following Figure 6 shows examples of actual data (“0”, “가” ) of numeric objects to be tested.

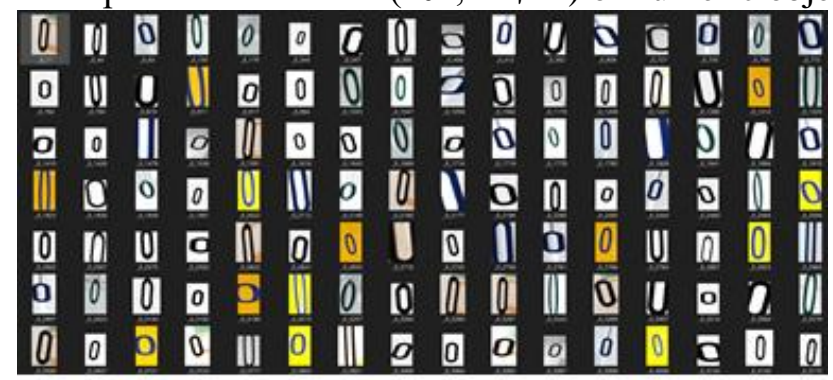

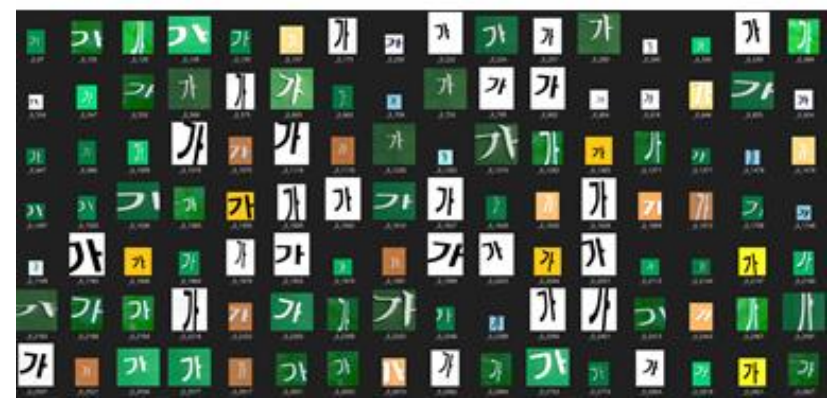

Fig. 5: 112 training data for Numbers (0) and Korean character (가). 


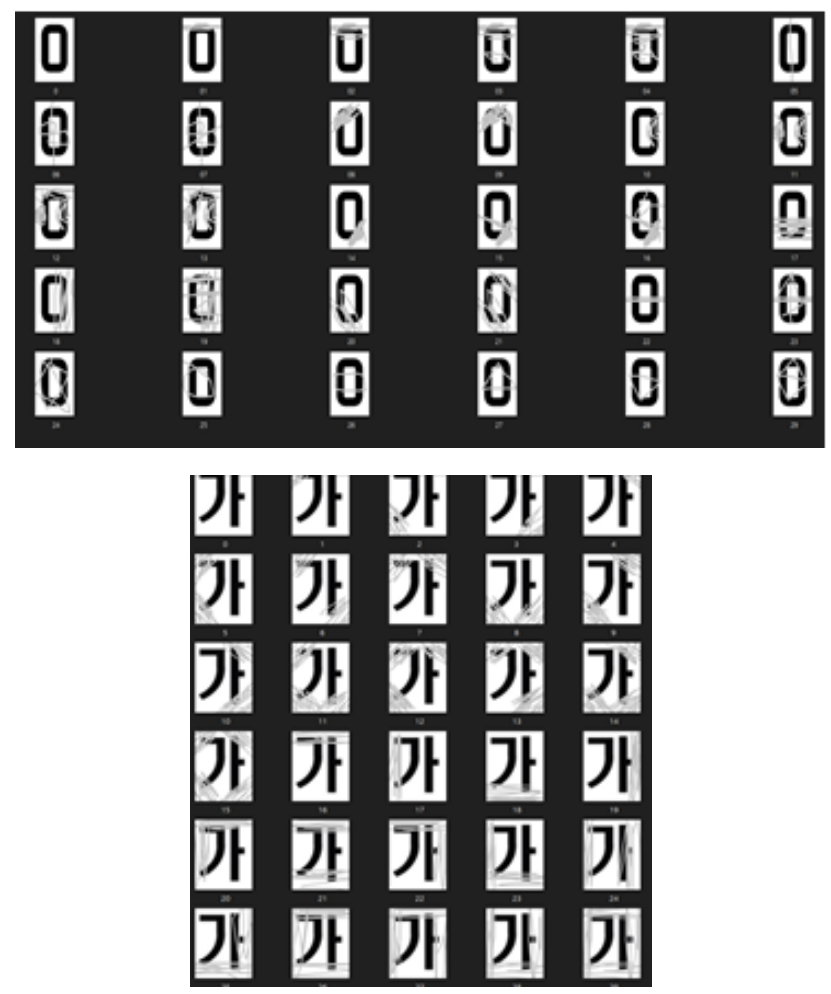

Fig. 6: Actual data of Number (0) and Korean character (가) to be tested.

\subsection{Experimental Results Analysis}

This experiment uses the proposed algorithm in 4.1, after completing CNN deep learning training to some extent through the training data of 4.2 (substituting 112 for each character), and then arbitrarily The results and analysis data for accuracy verification by substituting damaged characters (enter 30 data for each character) are shown in Figure 7, Figure 8.

One thing to note is that in the case of training data, the number of CNN deep learning training was performed 11 times for numbers and 10 times for Korean characters. In general, the more learning is done, the higher the accuracy. However, if too much learning is done, "overfitting" may occur, resulting in incorrect results, so it is necessary to limit the number of training sessions to an appropriate number.

Figure 7 shows the results and analysis of the numbers from 0 to 9 , and the above figure shows a simple matrix part and the lower part shows in more detail through graphic processing, and the $y$-axis is the provided element. And $x$ are the elements to match.

In the case of 0 (corresponding to the first line), 19 out of 30 answered 0 as the correct answer, 6 in the remaining answers, 6 answered with 6,4 answered with 8 , and 1 answered with 9 It was a dog. In other words, the correct answer rate with 
exactly 0 is expressed as $63.3 \%$, the incorrect answer rate is expressed as $36.7 \%$, and the remaining numbers (1 9) can also be checked in this way.

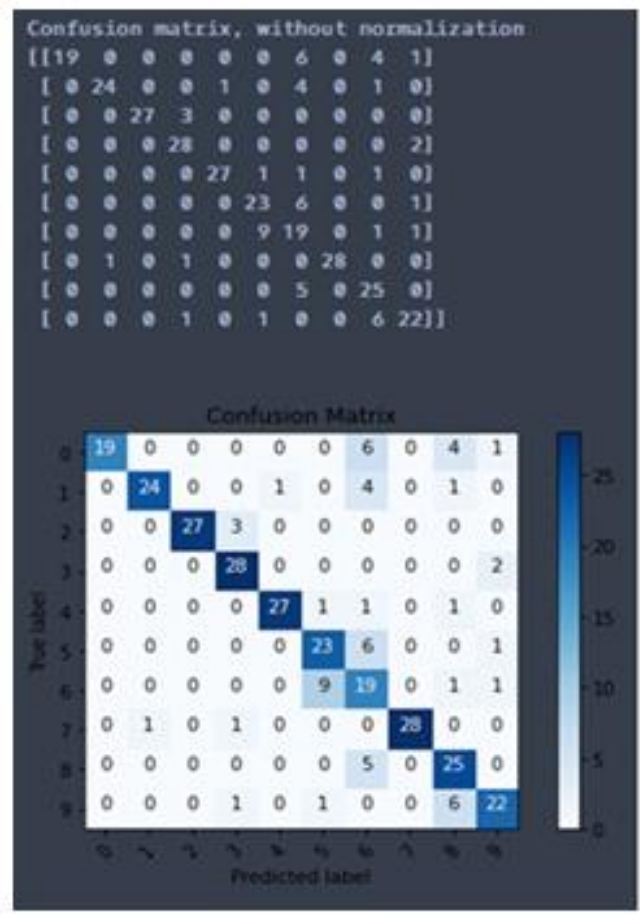

Fig. 7: Results of testing 10 numbers.

Figure 8 shows the results and analysis of the Hangul characters of "가" , “라” , "마" , and “자" . The above figure shows the simple matrix part and the lower part uses graphic processing. So, it is shown in more detail, and the y-axis is the provided element and $\mathrm{x}$ is the elements that need to be aligned.

In the case of "가" (corresponding to the first line), 29 out of 30 answered "가" , the correct answer, and 1 answered "라" in the remaining answers. In other words, the correct answer rate with exactly "가" is expressed as $96.6 \%$, the incorrect answer rate is expressed as $3.4 \%$, and in the case of the remaining letters("마”, “자”), the correct answer rate and the incorrect answer rate can be checked in this way. 


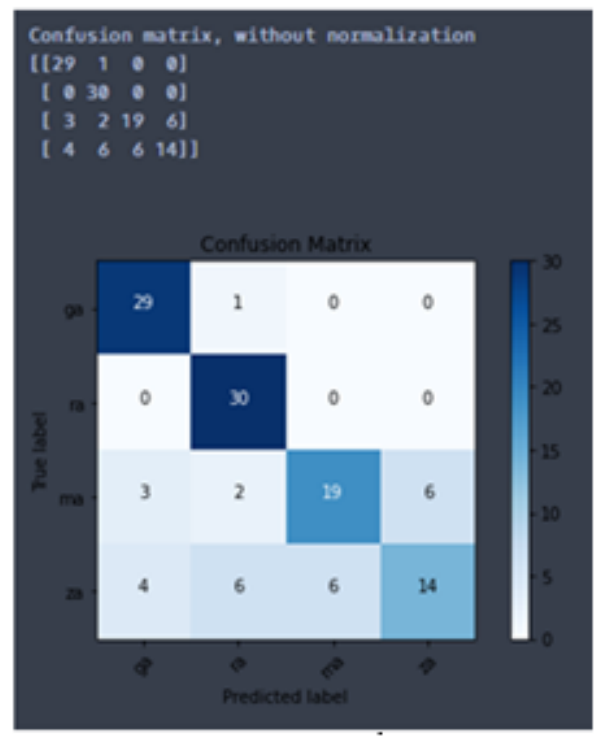

Fig. 8: Results of testing 4 Korean character.

Table 1: Recognition rate according to the number of test subjects of 10 number characters and the number of training participation

\begin{tabular}{|c|c|c|c|c|}
\hline $\begin{array}{c}\text { Number } \\
\text { Element }\end{array}$ & $\begin{array}{c}\text { Number of } \\
\text { Training } \\
\text { Subjects }\end{array}$ & $\begin{array}{c}\text { Number of } \\
\text { Training } \\
\text { Participation }\end{array}$ & $\begin{array}{c}\text { Number of } \\
\text { Training } \\
\text { Participation }\end{array}$ & $\begin{array}{c}\text { Number of } \\
\text { Test Subjects } \\
\text { Correct } \\
\text { Answer Rate } \\
(\%)\end{array}$ \\
\hline 0 & 112 & 11 & 30 & 63.3 \\
\hline 1 & 112 & 11 & 30 & 80 \\
\hline 2 & 112 & 11 & 30 & 90 \\
\hline 3 & 112 & 11 & 30 & 93 \\
\hline 4 & 112 & 11 & 30 & 90 \\
\hline 5 & 112 & 11 & 30 & 76.6 \\
\hline 6 & 112 & 11 & 30 & 63.3 \\
\hline 7 & 112 & 11 & 30 & 93 \\
\hline 8 & 112 & 11 & 30 & 83.3 \\
\hline 9 & 112 & 11 & 30 & 66.6 \\
\hline Average & 112 & 11 & 30 & 80 \\
\hline
\end{tabular}

After 11 training of training data (substituting 112 for each letter), experiment by substituting randomly damaged letters (entering 30 data for each letter) for the actual input data, 10 numbers and 4 letters. The analysis of the results (accuracy) of proceeding is as follows Table 1 and Table 2.

Analyzing the contents presented in the above result is as follows. That is, there is a slight difference for each number or Korean character, and there is a difference in recognition rate according to the degree of contamination and damage and uncertainty of each number data. The overall recognition rate for numeric characters 
is $80 \%$ and the recognition rate for Korean characters is $76.5 \%$. Perhaps, I think the recognition rate will be improved a little more if training data is inputted in more variety. However, unconditionally training data and training frequency do not increase the recognition rate, and research on optimal efficiency and overfitting should be continued.

Table 2: Recognition rate according to the number of test subjects of 4 Korean characters and the number of training participation

\begin{tabular}{|c|c|c|c|c|}
\hline $\begin{array}{c}\text { Number } \\
\text { Element }\end{array}$ & $\begin{array}{c}\text { Number of } \\
\text { Training } \\
\text { Subjects }\end{array}$ & $\begin{array}{c}\text { Number of } \\
\text { Training } \\
\text { Participation }\end{array}$ & $\begin{array}{c}\text { Number of } \\
\text { Training } \\
\text { Participation }\end{array}$ & $\begin{array}{c}\text { Number of } \\
\text { Test Subjects } \\
\text { Correct } \\
\text { Answer Rate } \\
(\%)\end{array}$ \\
\hline 가 & 112 & 10 & 30 & 96.6 \\
\hline 라 & 112 & 10 & 30 & 100 \\
\hline 마 & 112 & 10 & 30 & 63.3 \\
\hline 자 & 112 & 10 & 30 & 46.6 \\
\hline Average & 112 & 10 & 30 & 76.5 \\
\hline
\end{tabular}

\section{Conclusion}

As the standard of living increases due to the development of the industry, and the increase in the use of automobiles increases accordingly, various problems arise, and in particular, vehicle license plate automatic recognition system for parking lot access control and arrested vehicles according to the requirements of various fields there is an urgent need for improvement.

In this paper, a study was conducted on the recognition rate orientation of the existing recognition system, and in particular, the improvement of the recognition rate for numbers and Korean characters (Korean characters) that were unclear due to partial contamination or damage of Korean car license plates using CNN deep learning algorithms. The study was conducted.

For this experiment, 112 characters were prepared in various forms, each for training numbers and Korean characters, and 30 data were prepared for each actual test to randomly damage and contaminate. An experiment was conducted to determine (accuracy).

Also, for this experiment, research and experiments were conducted with the following 5 constraints. (1) A study on the recognition of automobile license plates in Korea. (2) A study on the recognition of license plates that are partially damaged, not normal characters, and are unclear by contamination. (3) The shape (degree of damage) and number of training data and actual data, and the number of training are arbitrarily determined for this experiment. (4) The application area of this study is an area where apartments and other vehicles are registered and managed. (5) The core of this study is a study on the recognition of unclear characters (numbers and Korean characters) using CNN deep learning algorithm. 
Analyzing the results of this experiment is as follows. That is, there is a slight difference for each number or Korean character, and there is a difference in recognition rate according to the degree of contamination and damage and uncertainty of each number data. The overall recognition rate for numeric characters is $80 \%$ and the recognition rate for Korean characters is $76.5 \%$. Perhaps, if the training data is input more diversely and trained, the recognition rate will be improved a little more. However, unconditionally training data and training frequency do not increase the recognition rate, and research on optimal efficiency and overfitting should be continued.

\section{References}

anaconda opensource. (2020). https://www.anaconda.com/products/individual.

Bae, J. (2020). Deep learning music genre classification system model improvement using generative adversarial networks (GAN). Journal of the Korea Institute of Information and Communication Engineering, 24(7), 842-848.

Choi, H. (1993). A Study on the tracking of car license plate coordinates in real time. Proceedings of the Joint Conference on Signal Processing, 6(1), 678-681.

Kang, S. W. (2020). Artificial intelligence / machine learning / deep learning concepts..http://blog.naver.com/jevida/221843366216, 2020.

Kim, J. W., and Choi, H. (1995). A study on character extraction algorithm for vehicle license plate recognition. The Korean Institute of Electrical Engineers, 7, 965-967.

Kim, Y. (2020). A study on the analysis of effective learning environment for vehicle license plate recognition based on CNN deep learning. from Late, Hannam University Master's Thesis.

Kim, Y. J., Hazra, D., Byun, Y., and Ahn, K. J. (2019). Old document restoration using super resolution GAN and semantic image inpainting. from ACM, WAIE 2019: International Workshop on Artificial Intelligence and Education Singapore.

Lee, G. S., Kim, Y. J., and Koh, D. K. (2020). Development of vehicle number recognition program based on deep learning algorithm. Journal of the Korean Cadastre Information Association, 22(2), 124-135. 
Liu, Z., and Kim, D. W. (2015). Digit recognition for vehicle license plate based on opened enclosure. Journal of Korea Institute of Information, Electronics, and Communication Technology, 8(6), 453-459.

Macfee, B. (2017). Learning content similarity for music recommendation. Journal of latex class files, 6(1), 1-2.

Naver Encyclopedia of Knowledge. (2020). Deep Learning-Thinking computer through learning (IT in terms of terms, Wonseok Oh). https://terms.naver.com/entry.nhn?docId=3578519\&cid=59088\&categoryId=59096.

Sai. (2020). Convolution neural network CNN basic concepts. https://blog.naver.com/mrp/221890752658.

Seo, M. J., and Kim, M. H. (2016). Data leakage detection system based on deep learning. International Journal of Reliable Information and Assurance, 4(2), 13-18.

Shang, C. J., and Brown, K. (1994). Principal feature-based texture classification with neural networks. Pattern Recognition, 27(5), 675-687.

Shon, Y. (2003). Printed numeric character recognition using fractal dimension and modified henon attractor. Journal of Korea Multimedia Society, 6(1), 89-96.

Tang Q. H., Seungho, Y., and Jaemin, K. (2019). Korean license plate recognition using CNN. Journal of IKEEE, 23(4), 222-227.

Weon, I. S., and Lee, S. G. (2019). Velocity-based object detection in dynamic environment using YOLO-based deep learning algorithm. International Journal of Multimedia and Ubiquitous Engineering, 14(1), 7-12.

Zhang, Z. (2018). A survey on deep learning techniques, applications and challenges. International Journal of Hybrid Information Technology, 11(1), 13-20. 